\title{
Graphite Oxide Preparation Following a Mechanochemical Green Route and Spontaneous Air Oxidation
}

G. Tarango-Rivero ${ }^{1}$, J.M. Mendoza-Duarte ${ }^{2}$, C.G. Garay-Reyes ${ }^{2}$, Ivanovich Estrada ${ }^{1}$ and R. MartínezSánchez ${ }^{2}$

${ }^{1}$ Centro de Investigacion en Materiales Avanzados, Chihuahua, Chihuahua, Mexico, ${ }^{2}$ Centro de Investigación en Materiales Avanzados (CIMAV), Laboratorio Nacional de Nanotecnología, Chihuahua, Chihuahua, México

* Corresponding author: ivanovich.estrada@cimav.edu.mx

The scope of the materials science is the study of macro properties of substances as a function of their morphological, microstructural, and chemical array, which are dependent on the preparation method and its basic control parameters. Although graphite (Gr) has been known since ancient times, Graphene has been taking into consideration for scientifics and technologists for a wide variety of modern applications due to their unique characteristics. Graphene can be prepared using a scalable and low-cost method based on the chemical oxidation of $\mathrm{Gr}$ to graphite oxide $(\mathrm{GrO})$, followed by a reduction process [1]. $\mathrm{GrO}$ is described as a high number of stacked layers of Gr decorated by oxygenated functional groups, which make it easier to exfoliate into Graphene monolayers [2]. Usually, GrO is synthesized by chemical oxidation techniques, but there are some inherent critical cons, such as longer processing time, poor particle size control, and toxic gas generation [3]. Furthermore, reaction byproducts contain harmful concentrations of acids and oxidizing agents that are a real threat to the environment [4]. High-energy ball milling (HEBM) is a solid-state technique that is used to mix, reduce, and change the shape of powder particles, refining the grains into nanoscale sizes increasing their surface area (SA). Thus, HEBM can be used to modify the layered structure of graphite without the solvents and chemicals necessity. This study deals with the preparation of $\mathrm{GrO}$ based on an alternative green route $\mathrm{Gr}$ (chemicals-free), based on HEBM and the spontaneous oxidation of the milled powders. $8 \mathrm{~g}$ of natural Gr flakes were processed in a Spex $8000 \mathrm{M}$ device using a ball to powder mass ratio of 5:1 in a steel vial for $8 \mathrm{~h}$. GrO was obtained simply by putting the fresh milled powder abruptly in contact with air (observing material incandescence). The characterization was performed through BET, SEM-EDS, and DRX.

Fig. 1a shows the adsorption-desorption isotherms of Gr samples; there is a considerable increase of SA from 1 to 500 units. HEBM induces the generation of highly active regions on the material surface, which increases the Gr chemical activity to atmospheric oxygen. The XRD pattern of Fig. 1b exhibits two main peaks of $\mathrm{Gr}$ around 26.59 and $54.67^{\circ}$. The (002) line corresponds to a plane spacing of $3.349 \AA$, indicating the hexagonal Gr structure. After HEBM, there are significant changes in their crystallinity (peaks become broader and intensity decreases from 600 to $20 \mathrm{~K}$ counts); this is related to crystalline deformation, grain refinement, and lattice distortion that occurs in the AB stacking sequence of Gr lattice due to processing. Although two remnants peaks can be seen in the $8 \mathrm{~h}$ sample, there is a formation of a broad peak located at a lower diffraction angle, which is usually related to GrO formation (observing an interlayer spacing of $0.67 \mathrm{~nm}$ ). Although there is not a consensus about $\mathrm{GrO} \mathrm{d}$-spacing, some a thickness from $0.57 \mathrm{~nm}$ to 0.79 $\mathrm{nm}$ is reported. In the images of Fig. 2 is evident that the Gr structure was effectively modified due to ball impacts during HEBM; there is a defoliation signal related to substantial comminution and layers sliding. The chemical analyses showed the presence of oxygen in the sample as a result of the partial combustion. 
Based on the experimental evidence, HEBM proved that it is a convenient and practical method for Gr partial oxidation avoiding the use of solvents and harmful chemicals, giving a product profoundly disordered
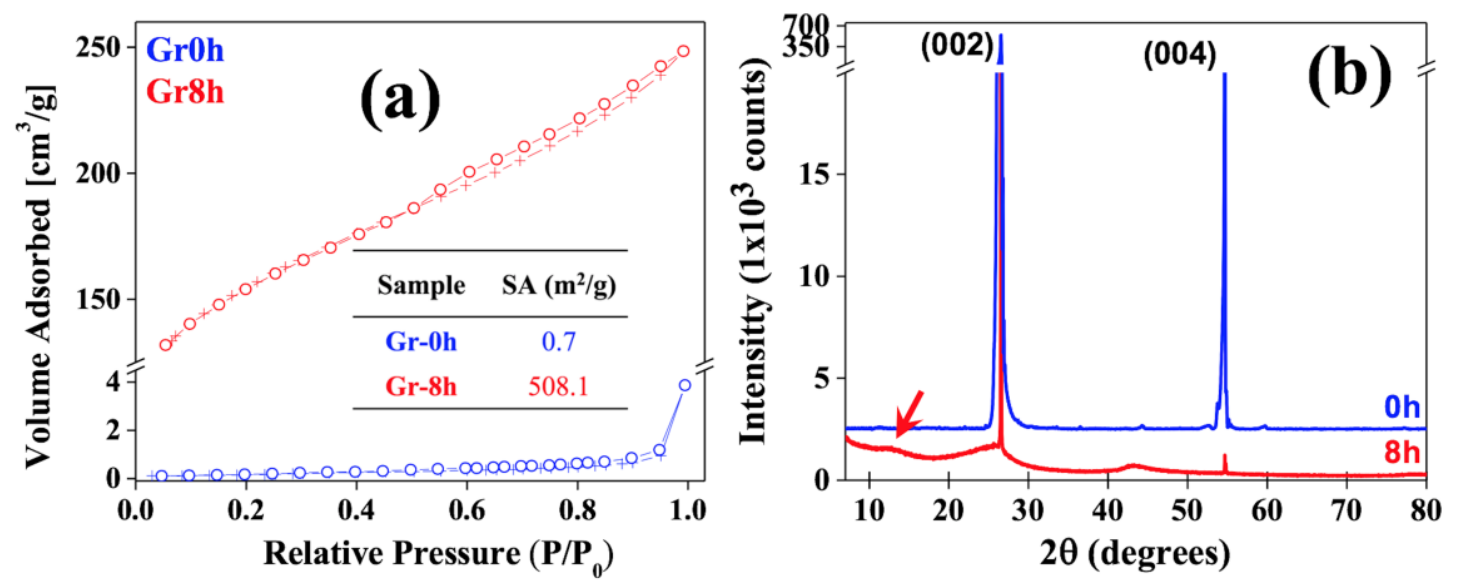

Figure 1. (a) Adsorption-desorption isotherms of Gr samples with surface area summary chart and (b) XRD patterns of original Gr (Oh) and HEBM'ed (8h) specimens.
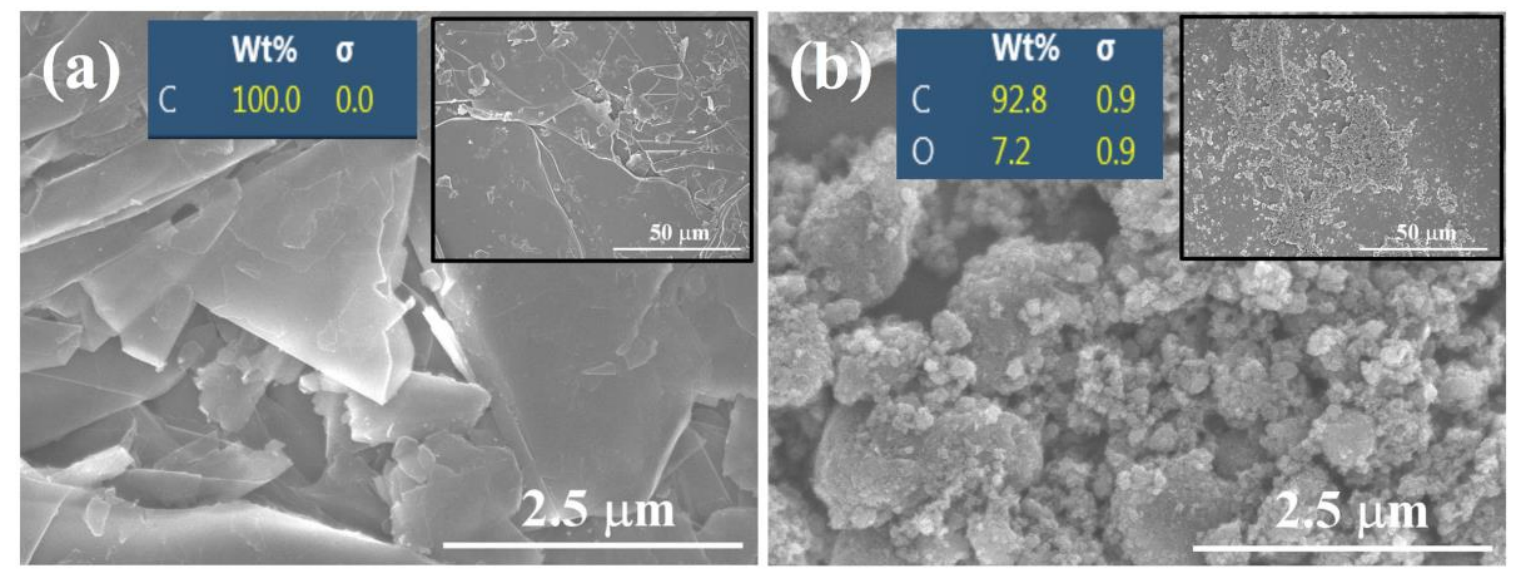

\section{References}

[1] C.H.A. Wong, O. Jankovsky, Z. Sofer, M. Pumera, Carbon 77 (2014), p. 508.

[2] K. Krishnamoorthy, M. Veerapandian, K. Yun, S.-J. Kim, Carbon 53 (2013), p. 38.

[3] G.K. Yogesh, E.P. Shuaib, P. Roopmani, M.B. Gumpu, U.M. Krishnan, D. Sastikumar, Diamond Rel. Mats. 104 (2020) 107733.

[4] B. Gurzęda, et al., App. Surf. Sci. 507 (2020), 145049. 